\title{
Vertical distraction osteogenesis of a free vascularized fibula flap in a recons- tructed hemimandible for mandibular reconstruction and optimization of the implant prosthetic rehabilitation. Report of a case
}

\author{
Gui-Youn Cho-Lee ${ }^{1}$, Luis Naval-Gías ${ }^{2}$, Pedro L. Martos-Díaz ${ }^{3}$, Raúl González-García ${ }^{4}$, Francisco J. Rodrí- \\ guez-Campo $^{2}$
}

\footnotetext{
${ }^{1}$ Resident. Department of Oral \& Maxillofacial Surgery. University Hospital La Princesa - Madrid, Spain

${ }^{2}$ Staff Surgeon. Department of Oral \& Maxillofacial Surgery. University Hospital La Princesa - Madrid, Spain

${ }^{3}$ Staff Surgeon. Department of Oral \& Maxillofacial Surgery. University Hospital Nuestra Señora de Candelaria. Carretera del Rosario. Santa Cruz de Tenerife, Spain

${ }^{4}$ Staff Surgeon. Department of Oral \& Maxillofacial Surgery. University Hospital Infanta Cristina. Badajoz, Spain Department of Oral \& Maxillofacial Surgery. (Head: Francisco J. Díaz-González). University Hospital La Princesa. Autónoma University. Madrid, Spain
}

Correspondence:

C/ Grañón 8, 1-3

28050 Madrid, Spain

gui-youncho@hotmail.com

Received: 13-12-2009 Accepted: $25-03-2010$

\begin{abstract}
Cho-Lee GY, Naval-Gías L, Martos-Díaz PL, González-García R, Rodríguez-Campo FJ. Vertical distraction osteogenesis of a free vascularized fibula flap in a recons- tructed hemimandible for mandibular reconstruction and optimization of the implant prosthetic rehabilitation. Report of a case Med Oral Patol Oral Cir Bucal. 2011 Jan 1;16 (1):e74-8. http://www.medicinaoral.com/medoralfree $01 / \mathrm{v} 16 \mathrm{i} 1 /$ medoralv16ilp74.pdf
\end{abstract}

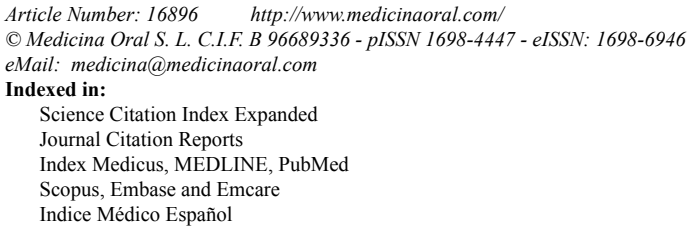

\begin{abstract}
Free vascularized fibular flap is considered the treatment of choice in mandibular reconstruction for extensive bone defects (over 6 centimeters) resulting from trauma, infections or tumor resections. But, when the reconstruction involves a dentate mandible, the fibula has the limit as it does not offer sufficient bone height to restore the alveolar arch up to the occlusal plane. Therefore, the deficiency in bone height makes implant placement impractical.

We report a case of vertical distraction osteogenesis of a free vascularized fibula flap used to reconstruct a hemimandible after resection of an odontogenic myxoma, for optimization of the implant prosthetic rehabilitation. The distraction device was applied intraorally. After 10 days of latency period, distraction protocol was performed at a distraction rate of $0.5 \mathrm{~mm}$ per day. A consolidation period of 3 months followed. Afterwards the distraction device was removed and 3 osseointegrated dental implants were placed in the distracted area. As a result, the vertical discrepancy between the fibula and the native hemimandible was corrected. The amount of vertical height achieved after distraction was 17 milimeters. The increase of vertical bone height was stable and enabled placement of dental implants without any complications. In conclusion, we consider that vertical distraction osteogenesis of free vascularized flaps is a reliable technique that optimizes implant positioning for ideal prosthetic rehabilitation, after mandibular reconstruction following tumor surgery.
\end{abstract}

Key words: Vertical distraction osteogenesis, free vascularized fibula flap, prosthetic rehabilitation. 


\section{Introduction}

The free vascularized fibula flap is considered the treatment of choice in mandibular reconstruction for extensive bone defects (over $6 \mathrm{~cm}$ ) resulting from trauma, infection or tumour resections (1). Since 1989, when Hidalgo first used the free vascularized fibula flap as a new method for reconstruction of the mandible, it has become an effective and safe procedure, giving excellent results both functionally and aesthetically (2).

The fibula flap presents many advantages such as adequate lenght (more than $20 \mathrm{~cm}$ of bone available), constant geometry, proper dimensions for implant placement, double periosteal and medullary blood supply allowing multiple osteotomies, correct contouring, an adequate pedicle lenght and low donor site morbidity. Bone thickness, height and its bicortical structure seem to be ideal for long-term implant prosthetic rehabilitation (3-5). But, because of its limited height (rarely more than $15 \mathrm{~mm}$ ) compared with the height of the mandible, vertical distance between the reconstructed segment and the occlusal plane can be substantial. This is a particular problem in the dentate mandible, especially when rehabilitation with dental implants is contemplated. Insufficient bone height leads to overloading of osseointegrated implants and jeopardizes the longevity of the prosthetic restoration (6-12).

Distraction osteogenesis is performed in cases of vertical resorption of edentulous jaws to improve bone volume for dental implant placement (alveolar distraction). This technique provides a very good quality of the neogenerated bone, with adequate characteristics for implant osseointegration (6).

This report presents a case of vertical distraction osteogenesis of a free vascularized fibula flap used to reconstruct a hemimandibular bone loss as a result of the surgical resection of an odontogenic myxoma, for improvement of implant prosthetic rehabilitation. The vertical discrepancy was completely corrected and 3 osseointegrated implants were placed in the distracted area. The prosthetic rehabilitation was carried out successfully with excellent functional and aesthetical results.

\section{Case report and surgical technique}

A 33-year old man was referred from another center with a large right hemimandibular mass that had experimented progressive growing. Wide surgical excision with a combined intraoral and extraoral approach was performed. Segmental mandibulectomy from the contralateral canine region to the ipsilateral coronoid process was perfomed. Condyle was preserved. Primary reconstruction my means of vascularized free fibula flap was achieved. The maximum height of the fibular bone was $14 \mathrm{~mm}$.

The reconstructed hemimandible was vertically defficient compared to the contralateral side, difficulting optimal

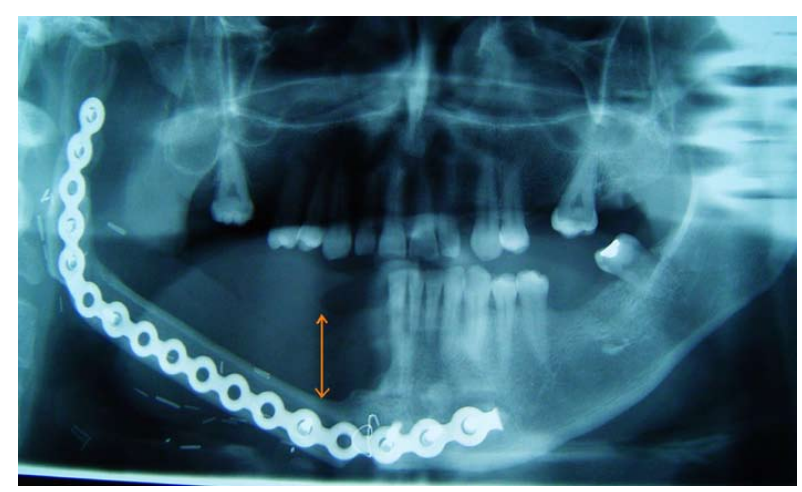

Fig. 1. Preoperative panoramic radiograph showing a 5-6 $\mathrm{cm}$ bone tumor with affectation of both mandibular walls extending form the second premolar to the middle of the right mandibular ramus.

implant placement. There was a height discrepancy of 17 $\mathrm{mm}$ (Fig. 1). For this reason, correction of the alveolar height was planned by using vertical distraction osteogenesis, six months after the mandibular reconstruction. The procedure was performed under general anesthesia. An intraoral incision in the buccal vestibule was performed, without lateral releasing incisions. Careful subperiosteal dissection was performed to obtain adequate visibility of the underlying bone, taking care to preserve the lingual mucoperiosteal attachment (Fig. 2A).

Preplatting and adjustment of the intraoral distractor (MODUS ARS 1.5; Medartis ${ }^{\circledR}$, Basel, Switzerland) was performed before starting the osteotomy. Osteotomies were performed with a sagital saw on the vestibular aspect of the fibula, under irrigation with sterile saline, and a greenstick fracture on the lingual side was achieved with chisels. The alveolar distractor was then applied and fixed by means of screws (Fig. 2B).

The vertical and the horizontal osteotomies were enlarged to allow movement of the segment with no interference. The device was then repositioned, stabilized, and temporarily activated to a distance of about $3 \mathrm{~mm}$ to ensure correct function during distraction. Finally, the osteotomized segment was repositioned at its initial position. Adequate contact between bony fragments and good hemostasis were obtained. Direct closure with 3/0 absorbable suture was performed. The distraction protocole included 10 days of latency period after surgery and a distraction rate of $0.5 \mathrm{~mm}$ per day. Orthopantomograms were taken weekly. The bone was distracted by about $17 \mathrm{~mm}$ in 34 days and the vertical discrepancy between the reconstructed hemimandible and the dentate contralateral hemimandible was corrected (Fig. 3A).

A consolidation period of 3 months followed. The distractor was left in place during the consolidation period to allow for maturation and consolidation of the neocallus formed between the 2 segments during distraction. Radiographic examination at the end of the consolidation 


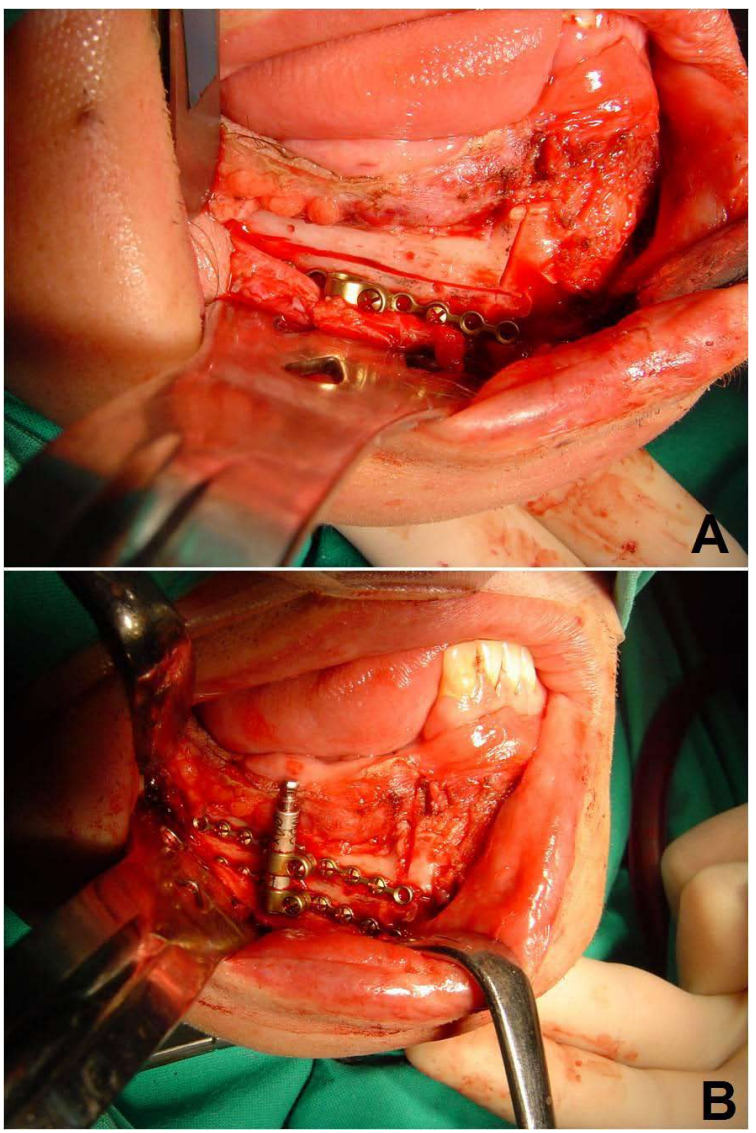

Fig. 2. Intraoperative views: intraoral alveolar distractor placement. A.Vestibular incision and subperiosteal dissection. Horizontal and vertical osteotomies completed. B.Alveolar intraoral distractor fixed to the cranial segment and basal bone of the fibular bone.
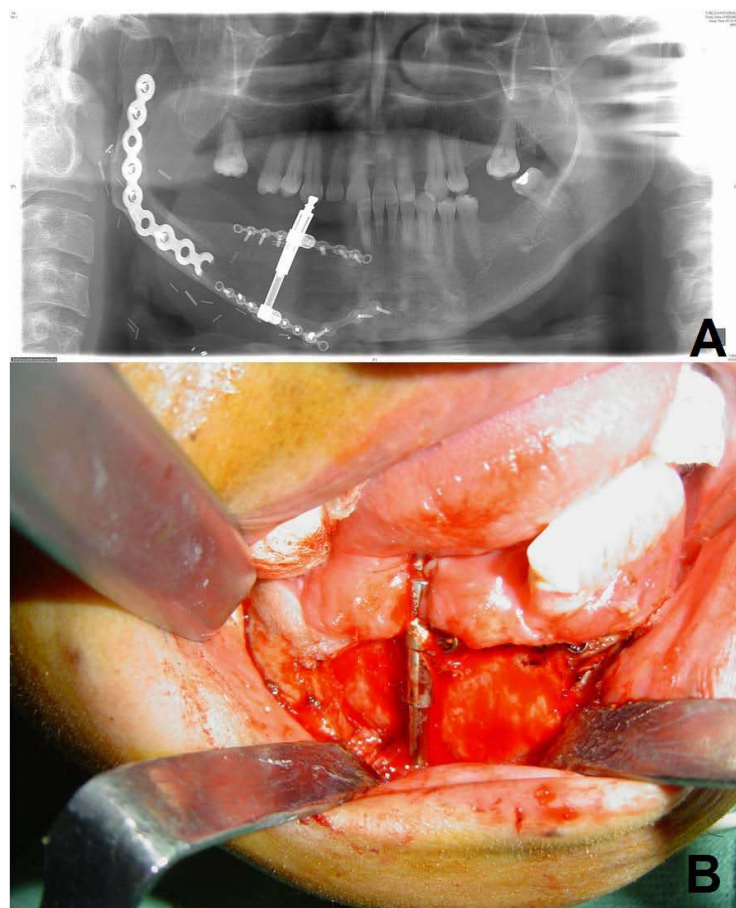

Fig. 3. A. Panoramic radiograph taken at the end of the consolidation period, showing complete filling of the space between the distracted segment and the lower border of the fibular bone by neogenerated tissue. B. Intraoperative view. Clinical situation at time of distractor removal and implant placement. The neogeneration of bone is clearly visible. Seventeen milimeters of newly generated bone were obtained. The quality of the bone was regarded macroscopically as excellent. The vertical discrepancy between the reconstructed hemimandible and the dentate contralateral hemimandible was corrected.
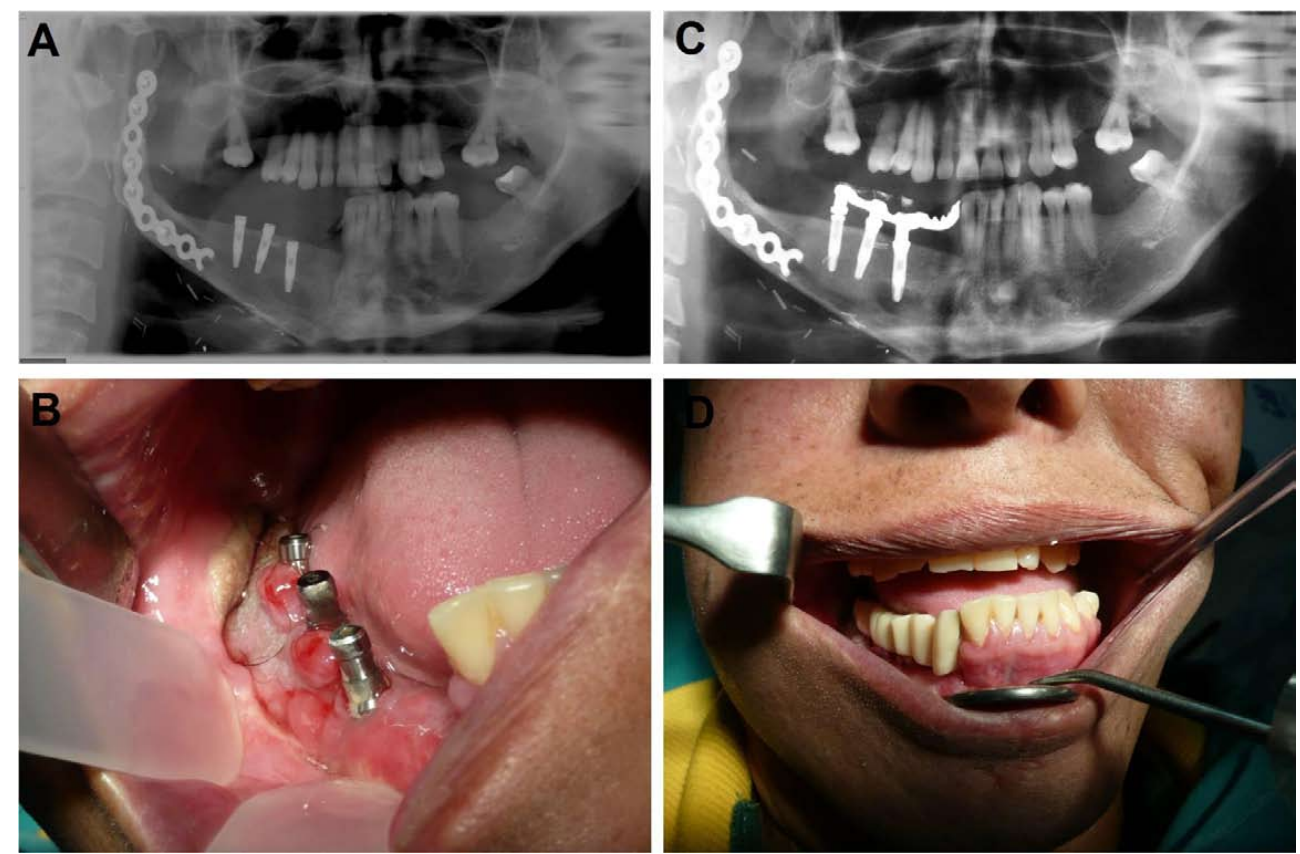

Fig. 4. A. Panoramic radiograph taken 3 months after the placement of 3 endosseous implants in the distracted area, showing an adequate boneto-implant interface. B. Intraoral view showing the implants placed in the distracted fibular bone. The intraoral soft tissues surrounding the reconstructed hemimandible have been elongated and adapted to the new situation. Three months after implants placement, little areas of granulation tissue appeared surrounding the implants, which were resected before prosthetic rehabilitation. C. Panoramic radiograph at the end of prosthetic rehabilitation. Implant-supported prosthesis. D. Intraoral view after prosthetic rehabilitation with an implant-supported prosthesis. 
period confirmed excellent ossification between the basal part of the fibula and the distracted segment (Fig. 3A).

The distractor was then removed, and good ossification was recognized in the distracted area. The quality of the new generated bone was regarded macroscopically as excellent (Fig. 3B).

After the device removal, 3 endosseous implants of 3,75 x $15 \mathrm{~mm}$ (MG Osseous ${ }^{\circledR}$, Mozo-Grau S.L. Valladolid, Spain) were placed in the distracted area (Fig. 4A). Primary stability was achieved for all the 3 implants (Fig. 4A, 4B). Postoperative period developed without complications. Prosthetic rehabilitation by means of implant-supported prosthesis was performed successfully 3 months after implants placement (Fig. 4C). Increased bone volume was stable during the follow-up period (24 months). Function and aesthetics were satisfactory for both the patient and the surgeon (Fig. 4D).

\section{Discussion}

Free fibula flap is reliable in providing both good functional and aesthetic results for reconstruction of mandibular bone defects. The main limitation of the fibula is the insufficient height (seldom more than $15 \mathrm{~mm}$ ) for the reconstruction of both the skeletal base and the alveolar ridge. This is especially evident in cases of partial loss of dentate mandibles. The discrepancy between the graft and the intact alveolar bone at the unaffected site does not allow prosthetic rehabilitation with an implantbased denture or a complete implant-borne prosthesis (6-12).

From a functional point of view, the implants need to support very long prosthetic suprastructures to reach the occlusal plane, with the risk of unfavourable bending moments and implant overload, which may jeopardize long-term implant survival. From an aesthetic point of view, very long crowns may represent unavoidable aesthetic problems $(6,7)$.

To overcome this problem, the fibula graft can be fixated at the level of the residual alveolar crest of the healthy side, but facial contour may be adversely affected (6-12). Alternatively, the double-barrel fibula flap technique can be used. This technique was described by Horiuchi et al. (1995) y Bähr et al. (1998) (13). Its limits are represented by the fact that in the case of large defects, the length of the bone segment may not be enough for duplication of the entire reconstructed part. Moreover, this method is technically demanding, with a higher risk of pedicle thombosis. The third possibility involves a further reconstructive procedure with a new revascularized flap or with an onlay bone graft fixed on the top of the first one. This solution is not usually recommended because of the further morbidity and is rarely accepted by a patient who has recently undergone a major surgical intervention (6-12). In these cases, vertical distraction osteogenesis of the fibular bone may be a solution to the problem (6). The development of the alveolar bone distraction device enabled us to perform vertical distraction of the fibular bone. The quality of the neogenerated bone is excellent with adequate characteristics for implant osseointegration (14). To our knowledge, only a few cases of vertical distraction osteogenesis of a fibula flap have been reported in the literature (6-12). Performing this technique there is no need of a second surgical procedure. It does not cause more morbidity. Furthermore, intraoral soft tissues surrounding the reconstructed hemimandible can also be elongated and adapted to the new situation. Vertical distraction osteogenesis is an excellent and reliable method to increase fibular bone height according to the individual local needs (6-12).

The optimal parameters for a successful vertical distraction such as latency, rate and rhythm have not been studied well. These parameters were mostly extrapolated from our own experience in mandibular distraction osteogenesis and the studies of craniofacial distraction osteogenesis (15). The latency period lasted 10 days, the distraction rate was $0.5 \mathrm{~mm}$ per day and the consolidation period lasted 3 months. The distraction rate of 0.5 $\mathrm{mm}$ per day is preferred by our team and it was maintained for this type of distraction. Some technical expedients were used: 1) It seems to be essential to maintain the lingual periosteal blood supply of the fibula during dissection. 2) The distraction vector was perpendicular to the major axis of the fibula.

Bone growth was clearly evident inside the distracted area. Seventeen milimeters of newly generated bone were obtained within 34 days. During mandibular reconstruction, good contour of the fibula usually requires multiple osteotomies. This procedure interrupts the medullary vessel and the periosteoum becomes the principal vascular supply $(12,13)$. This is why we waited for six months after fibula transfer before performing distraction osteogenesis. It may well be that this ensured complete bone regeneration.

During the distraction and the follow-up periods, radiographic examinations were performed by means of orthophantomograms that demonstrated the bone generation. Increased bone volume was stable during the follow-up period (24 months). Intraoral soft tissues surrounding the reconstructed hemimandible were elongated and adapted to the new situation without the tension and risk of exposure of the graft. Three months after implants placement, little areas of granulation tissue appeared surrounding the implants (Fig. 4B), which were resected before prosthetic rehabilitation. Moreover, the patient required a vestibuloplasty to optimize final functional and aesthetical results. A successful secondary vertical augmentation of the fibula flap was achieved in this case. Prosthetic rehabilitation by means of implant-supported prosthesis was performed success- 
fully and after 24 months of functional loading, no resorption was observed around the inserted implants. In this presented technique, there is no need for a second major procedure (new revascularized flap or autologous bone graft on top of the reconstructed mandibular segment). Moreover, the intraoral soft tissues surrounding the reconstructed mandible can be progressively elongated and adapted to the new situation without tension and risk of exposure of the graft. In this case, soft tissue stretched and covered the distracted region without any complication and vertical augmentation was not limited by the soft tissue.

Vertical distraction osteogenesis is an excellent and reliable method to increase fibular bone height according to the individual local needs, enabling a good prosthetic rehabilitation following the reconstruction of the mandible due to extensive bone defect resulting from tumour ablation. Furthermore, intraoral soft tissues surrounding the reconstructed hemimandible can also be elongated and adapted to the new situation.

Despite the limited experience, this modality of distraction osteogenesis seems to be a very interesting way to improve the final results of rehabilitation from a functional and aesthetic point of view. It should become a common procedure in the treatment of vertical alveolar ridge deficiency resulting from transplanting fibular bone for mandibular reconstruction following tumour resection.

\section{References}

References with links to Crossref - DOI

1. Cordeiro PG, Disa JJ, Hidalgo DA, Hu QY. Reconstruction of the mandible with osseous free flaps: a 10-year experience with 150 consecutive patients. Plast Reconstr Surg. 1999;104:1314-20.

2. Hidalgo DA. Fibula free flap: a new method of mandible reconstruction. Plast Reconstr Surg. 1989;84:71-9.

3. Hayter JP, Cawood JI. Oral rehabilitation with endosteal implants and free flaps. Int J Oral Maxillofac Surg. 1996;25:3-12.

4. Moscoso JF, Keller J, Genden E, Weinberg H, Biller HF, Buchbinder D, et al. Vascularized bone flaps in oromandibular reconstruction. A comparative anatomic study of bone stock from various donor sites to assess suitability for enosseous dental implants. Arch Otolaryngol Head Neck Surg. 1994;120:36-43.

5. Chang YM, Santamaria E, Wei FC, Chen HC, Chan CP, Shen YF, et al. Primary insertion of osseointegrated dental implants into fibula osteoseptocutaneous free flap for mandible reconstruction. Plast Reconstr Surg. 1998;102:680-8.

6. Chiapasco M, Brusati R, Galioto S. Distraction osteogenesis of a fibular revascularized flap for improvement of oral implant positioning in a tumor patient: a case report. J Oral Maxillofac Surg. 2000;58:1434-40.

7. Kürkcü M, Benlidayi ME, Kurtoğlu C, Kesiktaş E. Placement of implants in the mandible reconstructed with free vascularized fibula flap: comparison of 2 cases. Oral Surg Oral Med Oral Pathol Oral Radiol Endod. 2008;105:e36-40.

8. Schleier P, Hyckel P, Fried W, Beinemann J, Wurdinger J, Hinz $M$, et al. Vertical distraction of fibula transplant in a case of mandibular defect caused by shotgun injury. Int J Oral Maxillofac Surg. 2006;35:861-4.

9. Eski M, Turegun M, Deveci M, Gokce HS, Sengezer M. Vertical distraction osteogenesis of fibular bone flap in reconstructed mandible. Ann Plast Surg. 2006;57:631-6.
10. Ortakoglu K, Suer BT, Ozyigit A, Ozen T, Sencimen M. Vertical distraction osteogenesis of fibula transplant for mandibular reconstruction: a case report. Oral Surg Oral Med Oral Pathol Oral Radiol Endod. 2006;102:e8-11.

11. Klesper B, Lazar F, Siessegger M, Hidding J, Zöller JE. Vertical distraction osteogenesis of fibula transplants for mandibular reconstruction--a preliminary study. J Craniomaxillofac Surg. 2002;30:280-5.

12. Nocini PF, Wangerin K, Albanese M, Kretschmer W, Cortelazzi R. Vertical distraction of a free vascularized fibula flap in a reconstructed hemimandible: case report. J Craniomaxillofac Surg. 2000;28:20-4.

13. Bähr W, Stoll P, Wächter R. Use of the "double barrel" free vascularized fibula in mandibular reconstruction. J Oral Maxillofac Surg. 1998;56:38-44.

14. Chin M, Toth BA. Distraction osteogenesis in maxillofacial surgery using internal devices: review of five cases. J Oral Maxillofac Surg. 1996;54:45-53.

15. González-Garcia R, Rubio-Bueno P, Naval-Gías L, RodríguezCampo FJ, Escorial-Hernández V, Martos PL, et al. Internal distraction osteogenesis in mandibular reconstruction: clinical experience in 10 cases. Plast Reconstr Surg. 2008;121:563-75. 